\title{
ANNIHILATOR EQUIVALENCE OF TORSION-FREE ABELIAN GROUPS
}

\author{
P. SCHULTZ, C. VINSONHALER and W. J. WICKLESS
}

(Received 1 March 1990)

Communicated by R. Lidl

\begin{abstract}
We define an equivalence relation on the class of torsion-free abelian groups under which two groups are equivalent if every pure subgroup of one has a non-zero image in the other, and each has a non-zero image in every torsion-free factor of the other.

We study the closure properties of the equivalence classes, and the structural properties of the class of all equivalence classes. Finally we identify a class of groups which satisfy Krull-Schmidt and Jordan-Hölder properties with respect to the equivalence.
\end{abstract}

1991 Mathematics subject classification (Amer. Math. Soc.) $20 \mathrm{~K} 15$.

\section{Introduction}

Torsion-free abelian groups are notoriously difficult to classify, even up to quasi-isomorphism. In this paper we define an equivalence relation coarser than quasi-isomorphism on the class of all torsion-free abelian groups. We show that the equivalence classes form a structure for which the fundamental classification theorems of algebra are meaningful and frequently true. For example, Kaplansky's Test Problems have a positive solution for all equivalence classes, and the Krull-Schmidt and Jordan-Hölder Theorems hold for the equivalence classes of finite rank torsion-free abelian groups having no proper nilpotent endomorphisms.

(C) 1992 Australian Mathematical Society $0263-6115 / 92 \$ A 2.00+0.00$ 
Let $\mathscr{C}$ denote the class of all torsion-free abelian groups, hereafter simply called "groups". For any $A \in \mathscr{C}$, define the left annihilator of $A$ by ${ }^{\perp} A=$ $\{Y \in \mathscr{C}: \operatorname{Hom}(Y, A)=0\}$ and the right annihilator of $A$ by $A^{\perp}=\{X \in$ $\mathscr{C}: \operatorname{Hom}(A, X)=0\}$.

Define relations $<_{l},<_{r}$ and $\ll$ on $\mathscr{C}$ by $A<_{l} B$ if ${ }^{\perp} A \supseteq^{\perp} B, A<_{r} B$ if $A^{\perp} \supseteq B^{\perp}$, and $\ll=<_{l} \cap<_{r}$. These relations are clearly quasi-orders, and so induce equivalence relations $\sim_{l}, \sim_{r}$ and $\approx$ on $\mathscr{C}$ by $A \sim_{l} B$ if $A<_{l} B<_{l} A, A \sim_{r} B$ if $A<_{r} B<_{r} A$, and $\approx=\sim_{l} \cap \sim_{r}$. That is, for $A, B \in \mathscr{C}, A \approx B$ if and only if ${ }^{\perp} A={ }^{\perp} B$ and $A^{\perp}=B^{\perp}$.

The notion of annihilators dates back to [1]; they have been used repeatedly in the study of torsion theories. The relations $<_{r}$ and $\sim_{r}$ were defined, and the classification program initiated, in [4]. The dual relations $<_{l}$ and $\sim_{l}$ were defined in [5]. In the latter, Wickless studied especially the finite rank groups and showed that each such group $A$ has a pure subgroup $A_{l}$, called the left core of $A$, minimal with respect to $A \sim_{l} A_{l}$; and a factor group $A_{r}=A / K$, called the right core of $A$, such that $K$ is maximal with respect to $A \sim \sim_{r} A / K$.

The left core is defined as follows. If $\mathscr{E}(A)$ has no non-zero nilpotent endomorphisms, let $A_{l}=A$. Otherwise there exists $0=f^{2} \neq f \in \mathscr{E}(A)$. In this case let $A_{1}=\operatorname{ker} f$ and note that $A_{1}$ is a proper pure subgroup of $A$. Now consider $\mathscr{E}\left(A_{1}\right)$ and repeat this process to obtain, after a finite number of steps, a pure subgroup $A_{l}$ of $A$ such that $\mathscr{E}\left(A_{l}\right)$ has no non-zero nilpotent endomorphisms. It is not hard to check that $A \sim_{l} A_{1} \sim_{l} \cdots \sim_{l} A_{l}$.

To define the right core, we define a sequence $A^{i}$ of successive factor groups by $A^{0}=A$ and for $i \geq 0, A^{i+1}=A^{i} /\langle\operatorname{Im} f\rangle_{*}$, where $0=f^{2} \neq$ $f \in \mathscr{E}\left(A^{i}\right)$, and $\langle\operatorname{Im} f\rangle_{*}$ denotes the pure subgroup of $A^{i}$ generated by the image of $f$. The group $A_{r}$ is the first $A^{j}$ such that $\mathscr{E}\left(A^{j}\right)$ has no non-zero nilpotent endomorphisms.

In [5] it is shown that the left and right cores are unique up to quasiisomorphism and determine the $\sim_{l}$ and $\sim_{r}$ classes of $A$, in the sense that $A \sim_{l} B$ if and only if $A_{l}$ is quasi-isomorphic to $B_{l}$, and similarly for the right. These striking properties led the present authors to believe that a special rôle would be played by the $\approx$ classes of finite rank groups whose left and right cores are quasi-isomorphic. We call these "groups of core type"; they are investigated in Section 4.

The notation used is mostly standard. If $\mathscr{B}$ is a family of groups, $\oplus \mathscr{B}$ means the direct sum of the members of $\mathscr{B}$. We assume familiarity with the usual 'quasi'-concepts for finite rank groups. We denote quasi-equality and quasi-isomorphism by $\doteq$ and $\cong$ and the quasi-endomorphism ring of $G$ by $\mathbb{Q} \mathscr{E}(G)$. 


\section{Basic properties of the $\approx$ relation}

Let $\mathscr{A}$ be the class of $\approx$-equivalence classes $[A]$ for $A$ in $\mathscr{C}$, and let $\prec$ be the order induced on $\mathscr{A}$ by $\ll$.

Proposition 2.1. $\langle\mathscr{A}, \prec\rangle$ is a complete upper semi-lattice, where for all families $\mathscr{B}$ from $\mathscr{C}, \sup \{[A]: A \in \mathscr{B}\}=[\oplus \mathscr{B}]$.

Proof. We have a poset by definition, and it is routine to check that for all $C$ in $\mathscr{B}, C \ll \oplus \mathscr{B}$ and that if there exists $D \in \mathscr{C}$ such that for all $C \in \mathscr{B}, C \ll D$, then $\oplus \mathscr{B} \ll D$.

Proposition 2.2. Let $A \longrightarrow B \longrightarrow C$ be a short exact sequence in $\mathscr{C}$. Then $B \ll A \oplus C$, and $B \approx A \oplus C$ if and only if $A<_{r} B$ and $C<_{l} B$.

Proof. The definitions of $<_{l}$ and $<_{r}$ imply that $B \ll A \oplus C$ and that $A<_{l} B$ and $C<_{r} B$. Hence $A<_{r} B$ if and only if $A \ll B$, while $C<_{l} B$ if and only if $C \ll B$. The rest follows from Proposition 2.1.

Proposition 2.3. For all $A \in \mathscr{C},[A]$ is closed under isomorphism, arbitrary direct sums, extensions and subgroups of bounded index.

Proof. $[A]$ is obviously closed under isomorphism. Let $\mathscr{B}$ be a subset of [A]. Clearly $A \ll \oplus \mathscr{B}$ and by Proposition 2.1, $\oplus \mathscr{B} \ll A$. By Proposition $2.2,[A]$ is closed under extensions. Let $A \geq B \geq n A$ for some $n>0$. It is easy to check that ${ }^{\perp} A={ }^{\perp} B$ and $A^{\perp}=B^{\perp}$.

We shall see in Section 4 that in the finite rank case this result is best possible in the sense that if $\mathscr{E}(A)$ has no zero divisors, then the $\approx$-class $[A]$ is precisely the closure of $\{A\}$ under the four operations of Proposition 2.3. The next Proposition shows that the relation $\approx$ satisfies Kaplansky's Test Problems [3, Chapter 6].

Proposition 2.4. (1) Let $A, B, C$ and $D \in \mathscr{C}$ with $A \approx B \oplus C$ and $B \approx A \oplus D$. Then $A \approx B$.

(2) Let $A$ and $B \in \mathscr{C}$ with $A \oplus A \approx B \oplus B$. Then $A \approx B$. 
Proof. Both parts follow immediately from Proposition 2.1.

\section{Intrinsic criteria for $A \approx B$}

In order to apply the definition of $\approx$, it is essential to be able to recognize when the group structures of $A$ and $B$ imply $A \approx B$.

Proposition 3.1.

(1) $A<_{1} B$ if and only if for all non-zero pure subgroups $C$ of $A$, $\operatorname{Hom}(C, B) \neq 0$.

(2) $A<_{r} B$ if and only if for all torsion-free factor groups $D$ of $A$, $\operatorname{Hom}(B, D) \neq 0$.

Proof. The proof is a routine argument from the definitions of the relations $<_{l}$ and $<_{r}$.

Definition 3.2. (1) For any $A$ and $B$ in $\mathscr{C}$, define a smooth decreasing chain of pure subgroups of $A$ by $A^{0}[B]=A ; A^{1}[B]=\cap\{\operatorname{ker} f: f \in$ $\operatorname{Hom}(A, B)\} ; A^{\nu+1}[B]=\left(A^{\nu}[B]\right)^{1}[B] ;$ if $\nu$ is a limit ordinal, $A^{\nu}[B]=$ $\cap_{\mu<\nu} A^{\mu}[B]$. At each ordinal $\nu$, either $A^{\nu+1}[B]<A^{\nu}[B]$ or $A^{\nu+1}[B]=$ $A^{\nu}[B]$. Hence the chain eventually stabilizes.

The left $B$-length of $A$ is $\lambda=\lambda(A[B])=\inf \left\{\nu: A^{\nu}[B]=A^{\nu+1}[B]\right\}$. The $B$-radical of $A$ is $A[B]=A^{\lambda}[B]$.

(2) For any $A$ and $B$ in $\mathscr{C}$, define a smooth increasing chain of pure subgroups of $A$ by $A^{0}(B)=0 ; A^{1}(B)=\left\langle\operatorname{tr}_{B}(A)\right\rangle_{*}$ (i.e. the pure subgroup of $A$ generated by all homomorphic images of $B$ in $A) ; A^{\nu+1}(B) / A^{\nu}(B)=$ $\left(A / A^{\nu}(B)\right)^{1}(B)$; if $\nu$ is a limit ordinal, $A^{\nu}(B)=\cup_{\mu<\nu} A^{\mu}(B)$. As before, the chain stabilizes.

The right $B$-length of $A$ is $\rho=\rho(A(B))=\inf \left\{\nu: A^{\nu+1}(B)=A^{\nu}(B)\right\}$. The $B$-socle of $A$ is $A(B)=A^{\rho}(B)$.

Proposition 3.3.

(1) For all $A$ and $B \in \mathscr{C}, A<, B$ if and only if $A[B]=0$.

(2) For all $A$ and $B \in \mathscr{C}, A<_{r} B$ if and only if $A(B)=A$.

Proof. To simplify notation, put $\lambda=\lambda(A[B])$ and $\rho=\rho(A(B))$.

(1) $(\Rightarrow)$ For all $\nu$, if $A^{\nu}[B] \neq 0$, then by Proposition $3.1(1), A^{\nu+1}[B]$ is a proper subgroup of $A^{\nu}[B]$. Hence $A^{\lambda}[B]=0$. 
$(\Leftarrow)$ Let $X \in \mathscr{C}$ with $\operatorname{Hom}(X, B)=0$, and let $f \in \operatorname{Hom}(X, A)$. Then for all ordinals $\nu, X f \in A^{\nu+1}[B]$; otherwise there is a $g \in \operatorname{Hom}\left(A^{\nu}[B], B\right)$ with $0 \neq f \circ g \in \operatorname{Hom}(X, B)$. Hence $X f \in A[B]=0$, so $X \in{ }^{{ }^{A}} A$.

(2) $\Leftrightarrow$ Since $\operatorname{Hom}\left(B, A / A^{\rho}(B)\right)=0, \operatorname{Hom}\left(A, A / A^{\rho}(B)\right)=0$, so $A^{\rho}(B)=A$.

$(\Leftrightarrow)$ Let $Y \in \mathscr{C}$ with $\operatorname{Hom}(B, Y)=0$. Clearly $\operatorname{Hom}\left(A^{0}(B), Y\right)=0$; assume $\operatorname{Hom}\left(A^{\mu}(B), Y\right)=0$ for all $\mu<\nu$. Let $f \in \operatorname{Hom}\left(A^{\nu}(B), Y\right)$. Then, for all $\mu<\nu, f$ induces $\left.\bar{f} \in \operatorname{Hom} A^{\nu}(B) / A^{\mu}(B), Y\right)$. If $a \in A^{\nu}(B)$ with af $\neq 0$, note that $a \in A^{\mu+1}(B) \backslash A^{\mu}(B)$ for some $\mu+1 \leq \nu$. Then $0 \neq n(a+$ $\left.A^{\mu}(B)\right)=b g$ for some $n \in \mathbb{Z}, b \in B$ and $g \in \operatorname{Hom}\left(B, A^{\mu+1}(B) / A^{\mu}(B)\right)$. It follows that $0 \neq g \circ \bar{f} \in \operatorname{Hom}(B, Y)$, a contradiction, so $\operatorname{Hom}\left(A^{\nu}(B), Y\right)=$ 0 .

Hence $\operatorname{Hom}\left(A^{\rho}(B), Y\right)=\operatorname{Hom}(A, Y)=0$, so $Y \in A^{\perp}$.

\section{Groups of core type}

In this section we consider only groups of finite rank. Recall from the introduction the definitions of left and right core.

Definition 4.1. A group $A$ has core type if $A_{l} \cong A_{r}$.

A family of groups $\mathscr{B}=\left\{G_{1}, \ldots G_{r}\right\}$ for some positive integer $r$ is called a core system if each $\mathscr{E}\left(G_{j}\right)$ has no zero-divisors and each $\operatorname{Hom}\left(G_{i}, G_{j}\right)=0$ if $i \neq j$.

Note that a finite rank group has a division ring as quasi-endomorphism ring if and only if its endomorphism ring has no zero-divisors, [2, Vol II, $p$. 149].

Proposition 4.2. A group $A$ has core type if and only if $A \approx \oplus \mathscr{B}$ for some core system $\mathscr{B}$. In this case, the groups in $\mathscr{B}$ are unique up to quasiisomorphism.

Proof. Suppose $A$ is a group of core type and let $G=A_{l}$. Then $A \sim_{l} G$ and $A \sim_{r} \cong A_{r} G$, so $A \sim_{r} G$ as well. Thus $A \approx G$. Since $G$ is a finite rank group with no nilpotent endomorphisms, $G$ has a quasi-decomposition $G \doteq \oplus_{i \in[1, r]} G_{i}$ where for each $i \in[1, r], \mathbb{Q} \mathscr{E}\left(G_{i}\right)$ is a division ring and $\operatorname{Hom}\left(G_{i}, G_{j}\right)=0$ for $i \neq j$. Take $\mathscr{B}=\left\{G_{i}: i \in[1, r]\right\}$.

Conversely, if $A$ is a finite rank group with $A \approx G=\oplus \mathscr{B}$ for some core system $\mathscr{B}$, then $A_{l} \sim_{l} A \sim_{l} G$ and $A_{r} \sim_{r} A \sim_{r} G$. Now $A_{l}, A_{r}$ and $G$ have no non-zero nilpotent endomorphisms so by [5, Theorems 12 and 13 ,

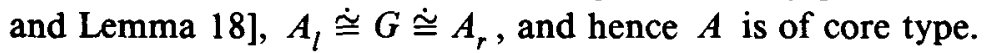


For uniqueness, suppose $A \approx G^{\prime}=\oplus \mathscr{B}^{\prime}$ for some other core system $\mathscr{B}^{\prime}$. Then, as above, $A_{l} \stackrel{\cong}{\cong} G^{\prime}$, so $G \stackrel{\cong}{\cong} G^{\prime}$. Since each group in a core system is strongly indecomposable, the result follows from the uniqueness of quasidecompositions.

Corollary 4.3. If $A$ has core type and $A \approx B$, then $B$ has core type.

We now use these results to prove a Krull-Schmidt Theorem for groups of core type, up to equivalence. First we need notions of indecomposability and uniqueness with respect to $\approx$. A naive approach is not sufficient, since for any group $A, A \approx A \oplus A$.

Definition 4.4. A group $G$ is $\approx$-indecomposable if whenever $A$ is a nonzero pure subgroup of $G$ with $G \approx A \oplus G / A$, then $A=G$.

The $\approx$-indecomposables are easily characterized.

Proposition 4.5. $G$ is $\approx$-indecomposable if and only if $\mathscr{E}(G)$ has no zero-divisors.

Proof. $(\Rightarrow)$ Let $G \doteq \oplus_{i \in[1, m]} A_{i}$ be a quasi-decomposition of $G$ into strongly indecomposable quasi-summands. Then by Proposition 2.3, $G \approx$ $\oplus_{i \in[1, m]} A_{i}$ so $m=1$ and $G$ is strongly indecomposable. Thus if $\mathscr{E}(G)$ has zero divisors, it has non-zero nil radical [2, Vol. II, p. 149] and hence contains a non-zero $f$ with $f^{2}=0$. Let $A=\langle G f\rangle_{*}$, a proper pure subgroup of $G$. Then $A \leq \operatorname{ker} f$, so $f$ induces a homomorphism of $G / A$ into $A$ whose image is full in $A$. Hence $G / A<_{l} A<_{l} G$ and $A<_{r} G / A<_{r} G$, so by Proposition $2.2, G \approx A \oplus G / A$, a contradiction.

$(\Leftarrow)$ Let $A$ be a non-zero pure subgroup of $G$ with $G \approx A \oplus G / A$. By Proposition 2.2, $A<_{r} G$ and $G / A<_{l} G$, so $\operatorname{Hom}(G, A) \neq 0$ and if $G / A \neq 0, \operatorname{Hom}(G / A, G) \neq 0$. Let $0 \neq f \in \mathscr{E}(G)$ with $G f \leq A$ and let $0 \neq g \in \mathscr{E}(G)$ with $A \leq \operatorname{ker} g$. Then $f \circ g=0$, a contradiction, so $A=G$.

COROLLARY 4.6. Let $G$ be a group of core type. Then there exists a core system $\mathscr{B}$ such that $G \approx \oplus \mathscr{B}$. The groups in $\mathscr{B}$ are unique up to quasiisomorphism and are $\approx$-indecomposable.

Proof. This follows from Propositions 4.2 and 4.5 and the definitions.

Corollary 4.6 is analogous to the Krull-Schmidt Theorem for finite rank groups up to quasi-isomorphism. We shall now prove an analogue of the Jordan-Hölder Theorem for finite groups.

Definition 4.7. Let $\mathscr{B}$ be a core system and let $G \doteq \oplus \mathscr{B}$. Let $H$ be a group. A left $G$-filtration for $H$ is a descending chain of pure subgroups 
$H=H_{0}>H_{1}>\cdots>H_{n}=0$ such that for $0 \leq i<n, H_{i} / H_{i+1}$ is quasi-isomorphic to a subgroup of some $G_{j(i)} \in \mathscr{B}$. We say $H_{i} / H_{i+1}$ is quasi-embedded in $G_{j(i)}$.

A $G$-filtration for $H$ is a left $G$-filtration such that each $H_{i} / H_{i+1}$ is quasiisomorphic to some $G_{j(i)}$.

Let $\mathscr{B}$ be a core system and let $G \doteq \oplus \mathscr{B}$. In [5] it was shown that for any group $H, H<_{l} G$ if and only if $H$ has a left $G$-filtration. The following lemma establishes an analogous result for the relation $\ll$ and $G$-filtrations.

LeMMA 4.8. Let $G \doteq \oplus \mathscr{B}$ for some core system $\mathscr{B}$. Let $0 \neq H$ be a group with $H \ll G$. Then $H$ has a G-filtration.

Proof. Let $\mathscr{B}=\left\{G_{1} \ldots G_{m}\right\}$. Since $H<_{l} G, H$ has a left $G$-filtration $H=H_{0}>H_{1}>\cdots>H_{n}=0$ with quasi-embeddings $\alpha_{i}: H_{i} / H_{i+1} \longrightarrow G_{j(i)}$ for $0 \leq i<n$ where $G_{j(i)} \in \mathscr{B}$.

The proof of the lemma is by induction on the rank $r$ of $H$. If $r=1$ then $n=1$ and $H=H_{0}$ is quasi-embedded in some $G_{j} \in \mathscr{B}$. Moreover since $H<_{r} G, \operatorname{Hom}(G, H) \neq 0$ so some $\operatorname{Hom}\left(G_{k}, H\right) \neq 0$. Since $\operatorname{Hom}\left(G_{k}, G_{j}\right)=0$ for $k \neq j$, it follows that $k=j$ and $\operatorname{Hom}\left(G_{j}, H\right) \neq 0$.

Let $0 \neq f \in \operatorname{Hom}\left(G_{j}, H\right)$ and let $\theta$ be a quasi-embedding of $H$ into $G_{j}$. Then $0 \neq f \theta \in \mathbb{Q} \mathscr{E}\left(G_{j}\right)$, a division algebra. Thus $f \theta$ is a quasi-invertible endomorphism of $G_{j}$ whose image is quasi-equal to $G_{j}$. Hence rank $G_{j}=1$ and $H \cong G_{j}$, so our result holds for $r=1$.

Now assume the result holds for all groups of rank smaller than $r$. Since $\operatorname{Hom}\left(G_{1}, G\right) \neq 0$ and $G<_{1} H, \operatorname{Hom}\left(G_{1}, H\right) \neq 0$. Let $0 \neq \beta: G_{1} \rightarrow H$. Choose $i$ so that $G_{1} \beta \leq H_{i}$, but $G_{1} \beta \not H_{i+1}$. Let $\delta$ be the composite map $G_{1} \stackrel{\beta}{\longrightarrow} H_{i} \longrightarrow H_{i} / H_{i+1} \stackrel{\alpha_{i}}{\longrightarrow} G_{j(i)}$.

Since $\delta \neq 0, j(i)=1$ and $\delta$ is invertible in $\mathbb{Q} \mathscr{E}\left(G_{1}\right)$. Thus $\beta$ is monic and $H_{i} \doteq G_{1} \beta \oplus H_{i+1}$. Note that $G_{1} \beta \cong G_{1}$ is quasi-pure in $H$ since $H_{i}$ is pure in $H$.

Let $K$ be the purification of $G_{1} \beta$ in $H$. For $t \leq i$ let $\bar{H}_{t}=H_{t} / K$ and for $t \geq i+1$ let $\bar{H}_{t}=\left(H_{t} \oplus K\right) / K$. Then it is easy to check that $\bar{H}=\bar{H}_{0}>\bar{H}_{1}>\cdots>\bar{H}_{i}=H_{i+1}>\cdots>H_{n}=0$ is a left $G$-filtration of $\bar{H}=H / K$. It follows that $\bar{H}<_{l} G$.

Clearly $H<_{r} H<_{r} G$ and rank $H<$ rank $H$ so inductively there exists a filtration of $H$ with each factor quasi-isomorphic to some $G_{j(i)}$. To complete the proof we recall that $K \doteq G_{1}$ and construct the obvious $G$-filtration for $H$.

THEOREM 4.9. Let $G=\oplus \mathscr{B}$ for some core system $\mathscr{B}$. Let $H$ be a group. 
Then $H \approx G$ if and only if

(1) there exists a quasi-embedding $\alpha: G \longrightarrow H$;

(2) there exists a quasi-epimorphism $\pi: H \longrightarrow G$;

(3) there exists a G-filtration for $H$.

Proof. $(\Leftarrow)$ Condition (1) implies $G<_{l} H$, Condition (2) implies $G<_{r}$ $H$ and Condition (3) implies that $H \ll G$.

$\Leftrightarrow$ Since $H \sim_{l} G, H_{l} \cong G_{l}=G$, so there is a quasi-embedding of $G$

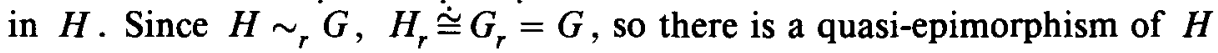
onto $G$.

The third condition follows from Lemma 4.8.

COROLLARY 4.10. Let $G$ be a group for which $\mathscr{E}(G)$ has no zero-divisors. Then $H \approx G$ if and only if $H$ has a filtration $H=H_{0}>H_{1}>\cdots>H_{n}=0$ with each $H_{i} / H_{i+1} \stackrel{\cong}{\cong}$.

The necessity for the assumption in Corollary 4.10 that $\mathscr{E}(G)$ have no zero-divisors is illustrated by the following example.

ExAmple. Let $A$ be a rank one group of nil type, and let $G_{1}$ and $G_{2}$ be non-quasi-isomorphic non-splitting extensions of $A$ by $A$. Then $G_{1} \approx G_{2} \approx$ $A$ but $G_{1}$ has no $G_{2}$-filtration.

CoRollaRy 4.11. Let $G$ be a group for which $\mathscr{E}(G)$ has no zero-divisors. Then the set of finite rank groups equivalent to $G$ is the closure of $\{G\}$ under the operations of quasi-isomorphism and extension.

For the next theorem, recall Definition 3.2 for $H^{j}[G]$ and $H^{j}(G)$.

THeOREM 4.12. Let $G$ and $H$ be groups such that $\mathscr{E}(G)$ has no zerodivisors. Then $H \approx G$ if and only iffor all $0 \leq j<\lambda(G[H]), H^{j}[G] / H^{j+1}[G]$

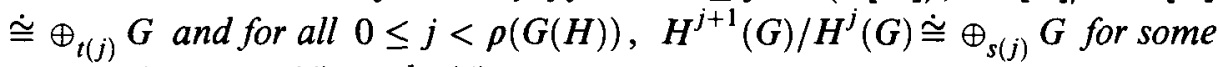
positive integers $t(j)$ and $s(j)$.

Proof. The sufficiency of the condition follows immediately from Corollary 4.10 .

For necessity, suppose $\mathscr{E}(G)$ has no zero-divisors and $H$ is a group with $H \approx G$. Then by Corollary $4.10, H$ has a filtration $H=H_{0}>H_{1}>\cdots>$ $H_{n}=0$ with each $H_{i} / H_{i+1} \stackrel{\cong}{\cong}$. The proof is by induction on $n$, and the result is clearly true if $n=1$. Let $n>1$ and assume the theorem holds for all groups having a shorter such filtration. To simplify notation, denote 
$U^{j}[G]$ by $U^{j}$ for any group $U$, and let $\oplus G$ denote an arbitrary finite direct sum of copies of $G$.

Let $\lambda$ be the left $G$-length of $H$ so $H^{\lambda}=0$, and let $0 \leq i<\lambda$ be such that $H_{n-1} \leq H^{i}$ but $H_{n-1} \notin H^{i+1}$. Regard $H^{i} / H^{i+1}$ as a subgroup of $\oplus G$. Since

$$
0 \neq W=\left(H_{n-1}+H^{i+1}\right) / H_{i+1} \leq H^{i} / H^{i+1} \leq \oplus G
$$

choose a projection $\pi$ onto some copy of $G$ such that $W \pi \neq 0$. Now $H_{n-1} \stackrel{\cong}{\cong} G$ and the composite map $H_{n-1} \hookrightarrow H^{i} \rightarrow H^{i} / H^{i+1} \stackrel{\pi}{\rightarrow} G$ is non-zero, so, since $\mathbb{Q} \mathscr{E}(G)$ is a division algebra, $H^{i} \doteq H_{n-1} \oplus X$ for some group $X$ containing $H^{i+1}$.

For any group $V$ with $H_{n-1} \leq V \leq H$, let $\bar{V}$ denote $V / H_{n-1}$. Note that $\bar{H} \approx G$ by Theorem 4.9 , so by the induction assumption, $\bar{H}^{j} / \bar{H}^{j+1} \cong \oplus G$ for all $j$. It now follows by induction on $j$ that for $0 \leq j \leq i$,

$$
(\bar{H})^{j}=\left(H / H_{n-1}\right)^{j}[G] \cong\left(H^{j}[G]\right) / H_{n-1}=\overline{\left(H^{j}\right)} .
$$

We prove that $H^{j} / H^{j+1} \cong \oplus G$ by considering three cases. First, let $0 \leq$ $j<i$. Then

$$
H^{j} / H^{j+1} \cong \overline{H^{j}} / \overline{H^{j+1}} \cong \bar{H}^{j} / \bar{H}^{j+1} \cong \oplus G .
$$

The first isomorphism follows from the second isomorphism theorem, the second from the paragraph above, and the quasi-isomorphism follows from the induction assumption. Thus our theorem holds for all $j$ with $0 \leq j<i$.

Next, let $i<j<\lambda$. Then $j=i+s$ for some $s>0$. For all $s>0$,

$$
H^{i+s}=\left(H^{i}\right)^{s} \stackrel{\cong}{\cong}\left(H_{n-1} \oplus X\right)^{s}=X^{s},
$$

since $H_{n-1} \doteq G$. Moreover, for $s \geq 0$,

$$
\bar{H}^{i+s}=\left(\bar{H}^{i}\right)^{s}=\overline{\left(H^{i}\right)^{s}} \stackrel{\cong}{\cong},
$$

since $\overline{H^{i}} \dot{\cong} X$. Thus for $s>0$,

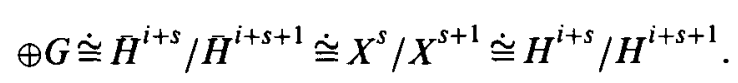

This proves the theorem for $j$ such that $i<j<\lambda$.

Finally,

$$
\begin{aligned}
& H^{i} / H^{i+1} \cong\left(H_{n-1} \oplus X\right) /\left(H_{n-1} \oplus X\right)^{1} \cong H_{n-1} \oplus X / X^{1} \\
& \dot{\cong} G \oplus\left(\bar{H}^{i} / \bar{H}^{i+1}\right) \doteq \oplus G .
\end{aligned}
$$

Thus, $H^{j}[G] / H^{j+1}[G] \cong \oplus G$ for $0 \leq j<\lambda$. A symmetric argument establishes the same claim for $H^{j+1}(G) / H^{j}(G)$ and the proof is complete. 
COROLlaRY 4.13. If $\mathscr{E}(G)$ has no zero-divisors, and $H \approx G$, then $\lambda(H[G])$ $=\rho(H(G))$.

Proof. Let $\lambda=\lambda(H[G])$ and $\rho=\rho(H(G))$. Then

$$
0=H^{\lambda}[G]<H^{\lambda-1}[G]<\cdots<H^{0}[G]=H,
$$

and

$$
0=H^{0}(G)<H^{1}(G)<\cdots<H^{\rho}(G)=H,
$$

where each factor is quasi-isomorphic to a direct sum of copies of $G$. Since $H / H^{\rho-1}(G) \doteq \oplus G$, it follows that $H^{1}[G] \leq H^{\rho-1}(G)$. Suppose we have shown that $H^{k}[G] \leq H^{\rho-k}(G)$. Then

$$
\begin{aligned}
H^{k}[G] /\left(H^{k}[G] \cap H^{\rho-k-1}(G)\right) & \cong\left(H^{k}[G]+H^{\rho-k-1}(G)\right) / H^{\rho-k-1}(G) \\
& \leq H^{\rho-k}(G) / H^{\rho-k-1}(G) .
\end{aligned}
$$

By Theorem 4.12, the last factor is quasi-equal to a direct sum of copies of $G$, so $H^{k+1}[G] \leq H^{\rho-k-1}(G)$. By induction, $H^{\rho}[G] \leq H^{0}(G)=0$, so $\lambda \leq \rho$. A symmetric argument shows that $\rho \leq \lambda$.

We close by showing that a Jordan-Hölder type theorem holds for the filtrations obtained in Lemma 4.8 and Theorem 4.9. The result may be of some independent interest.

TheOREM 4.14. Let $\mathscr{B}$ be a core system, let $H$ be a group, and let $H=$ $H_{0}>H_{1}>\cdots>H_{n+1}=0$ and $H=H_{0}^{\prime}>H_{1}^{\prime}>\cdots>H_{m+1}^{\prime}=0$ be filtrations

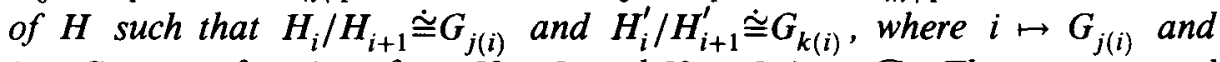
$i \mapsto G_{k(i)}$ are functions from $[0, n]$ and $[0, m]$ into $\mathscr{B}$. Then $n=m$ and there is a permutation $\sigma$ of $[0, n]$ such that $H_{i} / H_{i+1} \stackrel{\doteq}{\cong} H_{\sigma(i)}^{\prime} / H_{\sigma(i)+1}^{\prime}$ for all $i \in[0, n]$.

Proof. The proof is by induction on rank $H$, and the result is clear if rank $H=1$. Let rank $H=r>1$ and suppose the result holds for all groups of rank $<r$. Choose $i \geq 0$ minimal such that $H_{m}^{\prime} \leq H_{i}$ but $H_{m}^{\prime} \not H_{i+1}$. Arguing as in the proof of Lemma 4.8 we find $H_{i} \doteq H_{m}^{\prime} \oplus H_{i+1}$. Then

$$
H / H_{m}^{\prime}>H_{1}^{\prime} / H_{m}^{\prime}>\cdots>H_{m-1}^{\prime} / H_{m}^{\prime}
$$

and

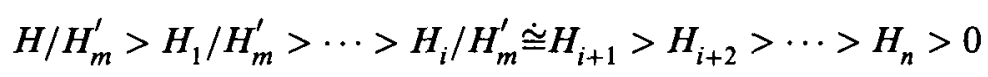

are filtrations of the group $H / H_{m}^{\prime}$ with factors in $\mathscr{B}$. By induction the factor groups in these new filtrations are quasi-isomorphic after rearrangement. But 
these factor groups coincide with those of the original filtrations except for the groups $H_{m}^{\prime}$ and $H_{i} / H_{i+1}$. Since $H_{i} / H_{i+1} \dot{\cong} H_{m}^{\prime}$, the proof is complete.

\section{References}

[1] S.C. Dickson, A Torsion Theory for Abelian Categories, Trans. Amer. Math. Soc. 121 (1966), 223-235.

[2] L. Fuchs, Infinite Abelian Groups, Vol. I and Vol. II, Academic Press, New York, 1970 and 1973.

[3] I. Kaplansky, Infinite Abelian Groups, University of Michigan Press, Ann Arbor, 1954.

[4] P. Schultz, Annihilator Classes of Torsion-free Abelian Groups, in Topics in Algebra, Lecture Notes in Mathematics 697, Springer-Verlag, Berlin, Heidelberg and New York, 1978.

[5] W. J. Wickless An equivalence relation for torsion-free groups of finite rank, preprint.

The University of Western Australia, Nedlands, Western Australia, 6009 Australia

University of Connecticut, Storrs, Connecticut, 06268

U.S.A.
University of Connecticut, Storrs, Connecticut, 06268

U.S.A. 